\title{
Experimental Determination of Air Drag on a Textile Yarn Struck Transversley by a High-Velocity Projectile
}

\author{
Jack C. Smith, Carl A. Fenstermaker, and Paul J. Shouse
}

(March 8, 1964)

\begin{abstract}
In recent research, rifle bullets having velocities in the range $100 \mathrm{~m} / \mathrm{s}$ to $500 \mathrm{~m} / \mathrm{s}$ have been shot at textile yarns, and flash photographs of the resulting configurations used as data for calculating stress-strain behavior. MeCrackin [J. Research NBS 66C, 317 (1962)] has shown how air drag may affect these data and calculations. MeCrackin's theory is tested here on a 93.3 tex nylon yarn and on 26.1 tex and 124.3 tex polyester yarns. The effect of yarn tex on air drag was found to agree with the theory. Curvature in the yarns resulting from air drag also agreed with theoretical predictions. Although curvature was noticeable in all tests, the effect of air drag on yarn tensile stress was negligible except for the 26.1 tex polyester yarn, in which case a small correction was necessary for accurate stress-strain calculations.
\end{abstract}

When a yarn or other filamentous material is struck transversely by a high-speed projectile, strain waves and waves of transverse motion are caused to propagate along the yarn away from the point of impact. In recent years, flash photographs of these yarn configurations have been obtained, and analyzed to provide stress-strain data $[1-5] .{ }^{1}$ In the analysis, it is desirable to estimate the effects of air drag on the yarn, and McCrackin [6] has recently shown how this may be done. In this paper McCrackin's theory is tested by applying it to some experimental data. The theory is then used to estimate the errors which would be incurred by neglecting the effect of air drag.

\section{Behavior of the Filament Without Air Drag}

Consider first the predicted behavior of the filament when air drag is neglected. Let the filament be struck transversely by a projectile moving with velocity $V$. The impact causes two waves of variable strain to propagate outward along the filament in each direction away from the point of impact. In the strained region between the two wave fronts, material of the filament is set into motion longitudinally toward the point of impact with velocity $W_{0}$ given by

$$
W_{0}=\frac{1}{\sqrt{m}} \int_{0}^{e_{0}} \sqrt{\frac{d T}{d e}} d e
$$

where $m$ is the mass per unit length of the unstrained filament, $e_{0}$ the maximum strain resulting from the impact, and $T$ the tension required to produce a strain $e$.

The inward flowing material forms itself into a wave shaped like a straight-sided wedge with the

\footnotetext{
1 Figures in brackets indicate the literature references at the end of this paper.
}

impacting projectile at the vertex. Material in this wave moves transversely with velocity $V$. The front of the transverse wave propagates along the filament with velocity $\lambda_{0}$ given by

$$
\lambda_{0}=\sqrt{\frac{T_{0}}{m\left(1+e_{0}\right)}}
$$

where $T_{0}$ is the tension in the filament resulting from the impact. The velocity $\lambda_{0}$ is expressed relative to the unstrained filament, i.e., with respect to a Lagrangian coordinate system.

The velocity actually observed, i.e., expressed relative to a coordinate system fixed to the laboratory, is $U_{0}$ given by

$$
U_{0}=\lambda_{0}\left(1+e_{0}\right)-W_{0} .
$$

The angle $\varphi_{0}$ between the original direction of the filament and the sloping side of the transverse wave is given by the relation

$$
\operatorname{Cos} \varphi_{0}=\frac{U_{0}}{\lambda_{0}}
$$

The theory is completed by an additional relation between the quantities just defined

$$
V^{2}=2 W_{0}\left(1+e_{0}\right) \lambda_{0}-W_{0}^{2} .
$$

If the effects of air drag are neglected, the above equations can be used to calculate $T_{0}$ as a function of $e_{0}$ from $U_{0}$ versus $V$ data obtained experimentally.

\section{Effect of Air Drag}

The force on the filament between the projectile and the transverse wave front due to air drag can be resolved into two components, one parallel and the other perpendicular to the filament. According to McCrackin, the effect of the parallel component is small and may be neglected. McCrackin also shows 
theoretically that the perpendicular component causes the filament to bend into the shape of a circular arc having a radius of curvature given by

$$
R=\frac{T}{F}
$$

where $T$ is the tensile force in the filament and $F$ is the normal force per unit length on the filament due to air drag. In addition the perpendicular component causes the strain and tension in the filament to increase from $e_{0}$ and $T_{0}$, respectively, by small amounts dependent upon the time elapsed after impact. The increases in strain and tension cause the transverse wave front to travel at increasing velocities slightly greater than $U_{0}$. Thus in actuality the strain, tension, and transverse wave front velocity are slightly greater than they would be if air drag were not present.

McCrackin shows that the strain $e$ in the filament when air drag is present is given approximately by

$$
e=e_{0}+a_{2} t^{2}+\mathrm{O}\left(t^{4}\right)
$$

and the tensile force by

$$
T=T_{0}+K a_{2} t^{2}+\mathrm{O}\left(t^{4}\right)
$$

where $t$ is the time elapsed after impact, $K$ is the slope of the tension-strain curve of the filament at strain $e_{0}$, and $\mathrm{O}\left(t^{4}\right)$ refers to terms of the order of $t^{4}$. The value of the coefficient $a_{2}$ as given by McCrackin is

$a_{2}=\frac{F^{2}}{4 T_{0}^{2}}$

$$
\left.\cdot \frac{\left(1+e_{0}\right)^{4} \lambda_{0}^{3}}{\left(4 \lambda_{0}+2 \sqrt{K / m}+k W_{0}\right)\left(1+e_{0}\right)+W_{0}\left(1-2 \sqrt{K / m} / \lambda_{0}\right.}\right)
$$

where $k$ is an abbreviation for $K / T_{0}$. To the same approximation, the velocity at which the transverse wave front travels is given by

$$
U=U_{0}+\left[\lambda_{0} k\left(1+e_{0}\right)+\lambda_{0}-2 \sqrt{K / m}\right] a_{2} t^{2} / 2+\mathrm{O}\left(t^{4}\right) .
$$

Equations (9) and (10) can be simplified slightly by use of some substitutions. The velocity, in Lagrangian coordinates, at which the strain $e_{0}$ propagates along the filament is given by

$$
C_{0}=\sqrt{\frac{1}{m}\left(\frac{d T}{d e}\right)_{e=e_{0}}}=\sqrt{\frac{\bar{K}}{m}} .
$$

Equations (2) and (11) together with the definition $k=K / T_{0}$ give the relationship

$$
k\left(1+e_{0}\right)=\frac{C_{0}^{2}}{\lambda_{0}^{2}} .
$$

By use of eqs (6), (11), and (12), eqs (9) and (10) can be expressed as follows:

$$
\begin{gathered}
a_{2}=\frac{1}{16 R^{2}} \frac{\left(1+e_{0}\right)^{3} \lambda_{0}^{2}}{1+\frac{C_{0}}{2 \lambda_{0}}+\frac{W_{0}}{4\left(1+e_{0}\right) \lambda_{0}}\left(\frac{C_{0}}{\lambda_{0}}-1\right)^{2}} \\
U=U_{0}+\frac{a_{2} \lambda_{0}}{2}\left(\frac{C_{0}}{\lambda_{0}}-1\right)^{2} t^{2}+\mathrm{O}\left(t^{4}\right) .
\end{gathered}
$$

\section{Experimental Tests}

In order to test McCrackin's theory on the effects of air drag, high velocity transverse impact tests were performed on three samples of textile yarns: a high-tenacity polyester (polyethylene terephthalate) yarn, $26.1 \mathrm{tex}^{2} 50$ filament; a high-tenacity polyester (polyethylene terephthalate) yarn, 124.3 tex, 250 filament; and a high-tenacity nylon (polyhexamethylene adipamide) yarn, 93.3 tex, 140 filament, $1 / 2 Z$ turns/in. manufacturer's twist. ${ }^{3}$

The yarns were suspended vertically under a light load of approximately $1 \mathrm{~g} / \mathrm{tex}$. The polyester yarns were given an additional twist of $2 Z$ turns/in. to prevent spreading of the filaments after impact. In the tests on nylon yarns some of the samples were twisted $1 / 2 Z$ and the others $1 \frac{1}{2} Z$ turns/in. The yarns were struck with rifle bullets at various velocities, and the resulting configurations during a series of short time intervals after impact were recorded by multimicroflash photography. A detailed description of the apparatus and technique has been given elsewhere [5].

The distance from the point of impact to either the top clamp or to the load affixed to the bottom of the yarn was $1 \mathrm{~m}$. Thus the front of the strain wave resulting from an impact traveled $100 \mathrm{~cm}$ to an end and returned approximately $80 \mathrm{~cm}$ before an interaction occurred between the reflected strain wave and the oncoming transverse wave. During the time required for the strain wave front to travel this distance, the velocity and the configuration of the transverse wave could be examined to determine the effects of air drag. For the polyester yarns this time was approximately $500 \mu \mathrm{s}$, and for the nylon yarn approximately $600 \mu \mathrm{s}$.

Figure 1 shows a specimen of the polyester yarn, 26.1 tex, impacted at $308 \mathrm{~m} / \mathrm{s}$. Fifteen successive flash pictures taken at $33.3 \mu$ s time intervals have been recorded as a multiple exposure. Only the left-hand side of the transverse wave is shown. The wave front in this case travels with a velocity $U$ of $450 \mathrm{~m} / \mathrm{s}$. Curvature due to air drag is quite apparent when a straight edge is laid alongside the wave for comparison.

\section{Results and Discussion}

Curvature due to air drag was observed in the polyester and nylon yarns at all of the transverse impact velocities. However, the wave front veloc-

${ }^{2}$ Yarn fineness or linear density is expressed in tex units. The tex value of a yarn is equal to the weight in grams of a $1000 \mathrm{~m}$ length of the yarn.

3 A yarn has " $Z$ " twist if, when held in a vertical position, the spirals or helices formed by the filaments incline in the same direction as the central portion of the letter " $Z$ ". 


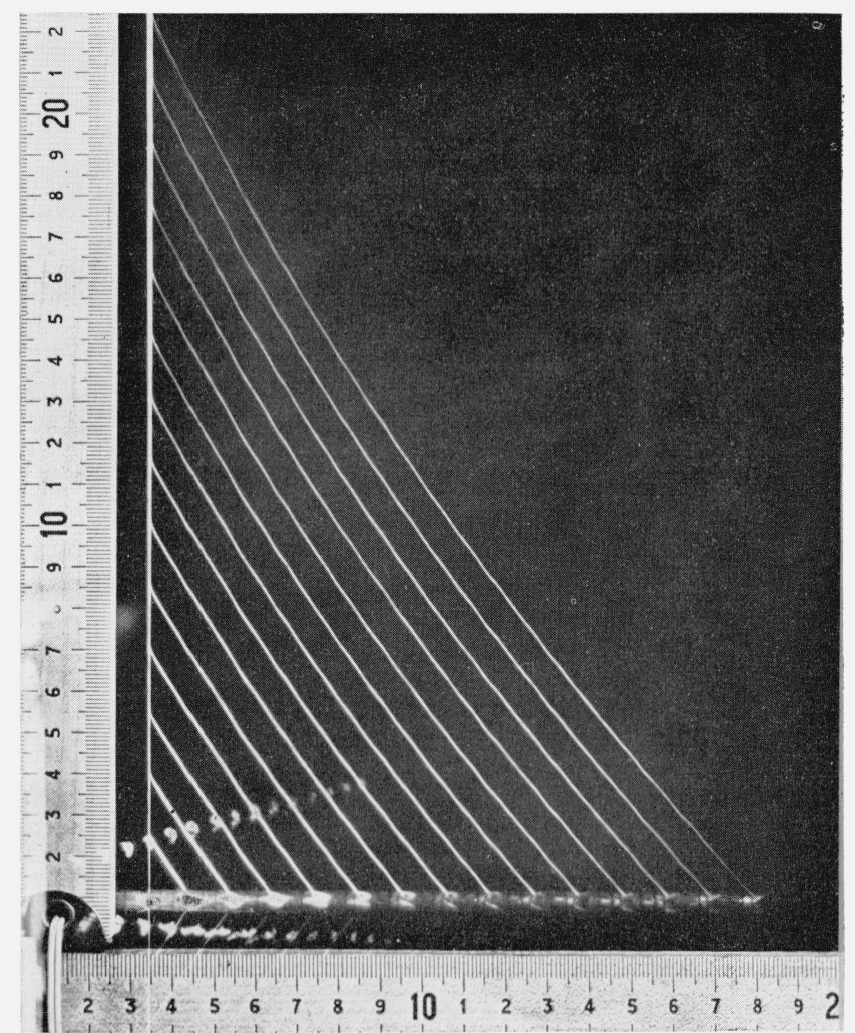

Figure 1. Effect of air drag on 26.1 tex polyester yarn. Transverse impact velocity was $308 \mathrm{~m} / \mathrm{s}$, and velocity of resulting transverse wave $450 \mathrm{~m} / \mathrm{s}$. Time between flashes was $33.3 \mu \mathrm{s}$. Radius of curvature due to air drag is $85 \mathrm{~cm}$ for the final yarn configuration shown.

ities $U$ of the transverse waves resulting from impact were found in each case to be constant; i.e., the wave front velocities showed no measurable increase with time. Thus the effect of air drag predicted by eq (14) was negligible for these yarn samples at the transverse impact velocities employed in the tests.

In the photographic enlargements the yarn configuration for each flash exposure could be closely approximated by a circular arc. To obtain the radius of curvature, a chord of length 2 a was drawn between two points on the yarn and the perpendicular distance $b$ from the midpoint of the chord to the varn measured. The radius of curvature is equal to $\left(a^{2}+b^{2}\right) / 2 b$.

In figure 2 the radius of curvature of the 26.1 tex polyester yarn is plotted as a function of the distance traveled (in laboratory coordinates) by the transverse wave front. For each transverse impact velocity, the radius of curvature is seen to increase toward a a plateau of constant values, as the transverse wave front advances along the yarn. This effect, which was not predicted by McCrackin's theory, may be due to the influence of different air drag forces at the impact and transverse wave front regions of the yarn. Air drag in the impact region is difficult to estimate, but air drag at the transverse wave front is small. Thus shortly after impact, when the length of yarn in the transverse wave is short, the air drag at the center of the transverse wave is greater than the air drag near the transverse wave front. The apparent radius of curvature calculated from measurements of the span $2 a$ and $\operatorname{sag} b$ would be small under these conditions. As the length of yarn in the transverse wave increases, the air drag becomes more uniformly distributed in the central region and the influence of the drag forces at the impact point and transverse wave front ends diminishes. Thus the apparent radius of curvature increases approaching the value predicted by McCrackin's theory, which did not consider end effects.

The radii of curvature are also observed to decrease as the velocity of impact is increased; i.e., curvature is more pronounced at the higher impact velocities, in agreement with McCrackin's theory. Similar behavior to that shown in figure 2 was observed for the nylon and 124.3 tex polyester yarns.

Tables 1, 2, and 3 present data obtained for the yarn samples. The values of transverse impact velocity $V$ and transverse wave front velocity $U$ obtained from measurements on the multimicroflash photographs for each test are given in the first two columns. The quantity $\varphi$ in the third column is the angle between the original position of the yarn and a straight line drawn from the wave front to the vertex of the transverse wave as determined from measurements on the photographs. $R$ is the radius of curvature obtained from measurements described previously.

Since air drag did not cause the wave front velocity to increase with time, its effect could be assumed negligible, and the $U, V$ data obtained from the tests could be used to calculate the tension strain behavior of the yarn for these impact conditions. The procedure, using theory neglecting the effects of air drag, has been described elsewhere [5]. The values of $T$ given in column 5 of the tables were determined by these calculations.

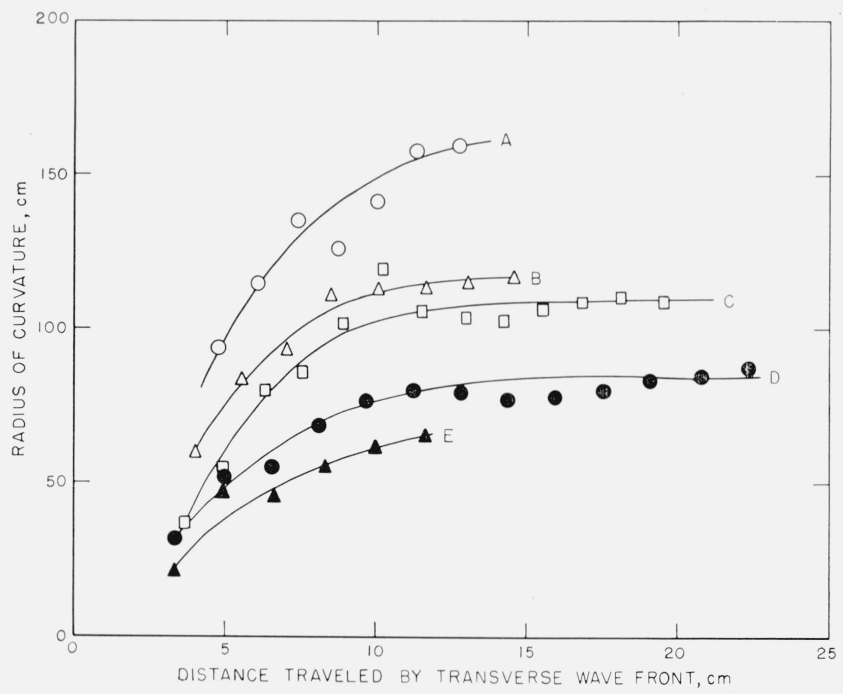

FiguRE 2. Plot of radius of curvature of 26.1 tex polyester yarn as a function of distance traveled by transverse wave front.

Impact velocities are: Curve A, $107 \mathrm{~m} / \mathrm{s} ; \mathrm{B}, 138 \mathrm{~m} / \mathrm{s} ; \mathrm{C}, 224 \mathrm{~m} / \mathrm{s} ; \mathrm{D}, 324 \mathrm{~m} / \mathrm{s}$ $\mathrm{E}, 381 \mathrm{~m} / \mathrm{s}$. 
Table 1. Data from impact tests on 26.1 tex high-tenacity polyester yarn

\begin{tabular}{|c|c|c|c|c|c|c|}
\hline$V$ & $U$ & $\operatorname{Cos} \varphi$ & $R$ & $T$ & $F$ & $F / V^{2} \cos ^{2} \phi$ \\
\hline $\begin{array}{l}m / s \\
107 \\
138 \\
169 \\
201 \\
224\end{array}$ & $\begin{array}{l}m / s \\
262 \\
300 \\
343 \\
377 \\
395\end{array}$ & $\begin{array}{r}0.926 \\
.910 \\
.897 \\
.883 \\
.868\end{array}$ & $\begin{array}{l}c m \\
170 \\
117 \\
120 \\
110 \\
110\end{array}$ & $\begin{array}{c}10^{6} \text { dyn } \\
0.201 \\
.283 \\
.368 \\
.458 \\
.530\end{array}$ & $\begin{array}{r}10^{3} \text { dyn/cm } \\
1.18 \\
2.42 \\
3.07 \\
4.17 \\
4.82\end{array}$ & $\begin{array}{r}10^{-5} \mathrm{~g} / \mathrm{cm}^{2} \\
1.20 \\
1.54 \\
1.34 \\
1.32 \\
1.27\end{array}$ \\
\hline $\begin{array}{l}273 \\
308 \\
324 \\
359 \\
381\end{array}$ & $\begin{array}{l}427 \\
450 \\
469 \\
498 \\
501\end{array}$ & $\begin{array}{l}.842 \\
.822 \\
.820 \\
.806 \\
.786\end{array}$ & $\begin{array}{l}93 \\
85 \\
85 \\
72 \\
70\end{array}$ & $\begin{array}{l}.673 \\
.799 \\
.818 \\
.921 \\
.984\end{array}$ & $\begin{array}{r}7.24 \\
9.41 \\
9.62 \\
12.79 \\
14.05\end{array}$ & $\begin{array}{l}1.37 \\
1.47 \\
1.36 \\
1.53 \\
1.57\end{array}$ \\
\hline
\end{tabular}

TABLE 2. Data from impact tests on 124.3 tex high-tenacity polyester yarn

\begin{tabular}{|c|c|c|c|c|c|c|}
\hline$V$ & $U$ & $\operatorname{Cos} \varphi$ & $R$ & $T$ & $F$ & $F / V^{2} \cos ^{2} \varphi$ \\
\hline $\mathrm{m} / \mathrm{s}$ & $\mathrm{m} / \mathrm{s}$ & & $\mathrm{cm}$ & $10^{6} d y n$ & $10^{3} \mathrm{dyn} / \mathrm{cm}$ & $10^{-5} \mathrm{~g} / \mathrm{cm}^{2}$ \\
\hline $\begin{array}{l}103 \\
210 \\
300 \\
342 \\
389 \\
408\end{array}$ & $\begin{array}{l}249 \\
380 \\
434 \\
447 \\
473 \\
485\end{array}$ & $\begin{array}{r}0.930 \\
.875 \\
.826 \\
.807 \\
.774 \\
.770\end{array}$ & $\begin{array}{l}325 \\
250 \\
190 \\
180 \\
150 \\
150\end{array}$ & $\begin{array}{l}0.93 \\
2.26 \\
3.28 \\
3.79 \\
4.32 \\
4.72\end{array}$ & $\begin{array}{r}2.86 \\
9.04 \\
17.28 \\
21.05 \\
29.54 \\
31.4\end{array}$ & $\begin{array}{l}3.13 \\
2.68 \\
2.81 \\
2.76 \\
3.26 \\
\text { 3. } 18\end{array}$ \\
\hline
\end{tabular}

TABLE 3.-Data from impact tests on 93.3 tex high-tenacity nylon yarn

\begin{tabular}{l|l|l|l|l|l|l}
\hline \hline$V$ & $U$ & $\operatorname{Cos} \varphi$ & $R$ & $T$ & $F$ & $F / V^{2} \cos ^{2} \varphi$ \\
\hline
\end{tabular}

Additional twist $1 / 2 Z$ turns/in.

\begin{tabular}{r|r|r|r|r|r|r}
\hline$m / s$ & $m / s$ & & \multicolumn{1}{c|}{$c m$} & $10^{6} \mathrm{dyn}$ & $10^{3} \mathrm{dyn} / \mathrm{cm}$ & $10^{-5} \mathrm{~g} / \mathrm{cm}^{2}$ \\
184 & 323 & 0.867 & 150 & 1.28 & 8.50 & 3.33 \\
189 & 323 & .864 & 145 & 1.29 & 8.91 & 3.35 \\
\hline
\end{tabular}

Additional twist $1 \frac{1}{2} Z Z$ turns/in.

\begin{tabular}{l|l|r|r|r|r|r}
\hline 306 & 427 & 0.810 & 147 & 2.39 & 16.28 & 2.65 \\
395 & 475 & .772 & 137 & 3.42 & 24.92 & 2.68 \\
\hline
\end{tabular}

The transverse force per unit length of yarn due to air drag was calculated from the values of $R$ and $T$ using eq (6). This quantity $F$ was tabulated in column 6. The tabulated values of $T$ and $F$ give the forces to which a yarn is subjected in a transverse impact test, and in this way add to an intuitive understanding of the process. For instance, it is seen from table 1 that the 26.1 tex polyester yarn impacted at a velocity $V$ of $381 \mathrm{~m} / \mathrm{s}$ is subjected to a tensile force of $0.984 \times 10^{6} \mathrm{dyn}(1.004 \mathrm{~kg})$ and a transverse air drag of $14.05 \times 10^{3} \mathrm{dyn} / \mathrm{cm}(14.34$ $\mathrm{g} / \mathrm{cm})$.

According to aerodynamical theory, the force per unit length $F$ due to air drag is given by $[6,7,8]$

$$
F=\frac{1}{2} \rho d C_{D} V^{2} \cos ^{2} \varphi
$$

where $\rho$ is the density of the air, $d$ the diameter of the yarn, and $C_{D}$ a dimensionless drag coefficient which varies slowly with Reynolds number. For the yarn diameters and impact velocities encountered in these tests Reynolds number has values between $2 \times 10^{3}$ and $2 \times 10^{4}$, and over this range of values the dimensionless drag coefficient is almost constant. According to data tabulated by Morrison and Ingle [7], $C_{D}$ for circular cross sections increases from 1.0 to 1.1 as Reynolds number increases from $1 \times 10^{4}$ to $2 \times 10^{4}$. McCrackin, using data given by Hoerner [8], chose a value for $C_{D}$ of 0.99 as applicable to textile yarns impacted transversely at velocities between $100 \mathrm{~m} / \mathrm{s}$ and $600 \mathrm{~m} / \mathrm{s}$.

From the discussion above it is seen that the quantity $1 / 2 \rho d C_{D}=F / V^{2} \cos ^{2} \varphi$ should be a constant for a yarn of given diameter. This quantity is tabulated in column 8 of the tables and is reasonably constant over the range of impact velocities used in the tests. Thus the values of $F$ determined using eq (6) are proportional to $V^{2} \cos ^{2} \varphi$ as predicted by eq (15). It is interesting to note that the values of $F / V^{2} \cos ^{2} \varphi$ given in table 3 for nylon yarn are lower for the samples having a $1 \frac{1}{2} \quad Z$ turns/in. twist than those for samples having only a $1 / 2 \quad Z$ twist. Evidently the more highly twisted samples have a smaller effective diameter, and hence a smaller value of $1 / 2 \rho d C_{D}$.

If $C_{D}$ is taken as 0.99 and $\rho$ as $1.20 \times 10^{-3} \mathrm{~g} / \mathrm{cm}^{3}$ (density of air at $20^{\circ} \mathrm{C}$ ) the quantity $F / V^{2} \cos ^{2} \varphi$ becomes equal to $5.94 \times 10^{-4} d$. The average value of $F / V^{2} \cos ^{2} \varphi$ for the 26.1 tex polyester yarn is $1.40 \times 10^{-5} \mathrm{~g} / \mathrm{cm}^{2}$ so that the "effective" diameter of the yarn becomes $1.40 \times 10^{-5} / 5.94 \times 10^{-4}=0.024$ $\mathrm{cm}$. If, however, the yarn is assumed to be a solid cylinder of polyethylene terephthalate of density $1.38 \mathrm{~g} / \mathrm{cm}^{3}$ [9], its diameter would be $0.015 \mathrm{~cm}$. Since there is some looseness to the packing of the yarn filaments, the two values of diameter just computed are in good agreement. Similarly, the average value of $F / V^{2} \cos ^{2} \varphi$ for the 124.3 tex polyester yarn is $2.97 \times 10^{-5} \mathrm{~g} / \mathrm{cm}^{2}$, from which a yarn diameter of $0.050 \mathrm{~cm}$ is derived. The diameter calculated from the density is $0.034 \mathrm{~cm}$, which is in good agreement. Average values of $F / V^{2} \cos ^{2} \varphi$ for the nylon yarn are $3.34 \times 10^{-5} \mathrm{~g} / \mathrm{cm}^{2}$ for the sample twisted $1 / 2 Z$ turns/in. and $2.66 \times 10^{-5} \mathrm{~g} / \mathrm{cm}^{2}$ for the sample twisted $1 \frac{1 / 2}{Z}$ turns/in. giving diameters of $0.056 \mathrm{~cm}$ and $0.045 \mathrm{~cm}$, respectively. The diameter calculated using a density for polyhexamethylene adipamide of 1.14 [9] is $0.033 \mathrm{~cm}$, which gives reasonable agreement.

The increase $\Delta U$ in transverse wave front velocity caused by air drag can be found from the expression

$$
\Delta U=\frac{1}{32 R^{2}} \frac{\left(1+e_{0}\right)^{3} \lambda_{0}^{3}\left(\frac{C_{0}}{\lambda_{0}}-1\right)^{2} t^{2}}{1+\frac{C_{0}}{2 \lambda_{0}}+\frac{W_{0}}{4\left(1+e_{0}\right) \lambda_{0}}\left(\frac{C_{0}}{\lambda}-1\right)^{2}}
$$

which is derived from eqs (13) and (14) given previously. Values of $\Delta U$ at times $200 \mu \mathrm{s}$ and $500 \mu$ s after impact at velocities between $100 \mathrm{~m} / \mathrm{s}$ and $500 \mathrm{~m} / \mathrm{s}$ are given for the three yarn samples in table 4. In order to compute these values of $\Delta U$, the experimentally obtained $U$ versus $V$ data uncorrected for air drag were used to calculate 
the stress-strain curve, the associated velocities $\lambda$, $W$, and $C$, and the angle of inclination $\varphi$. Additional data on the nylon and 124.3 tex polyester yarns were available from other experiments [5]. Values of $F$ were calculated from the average values of $F / V^{2} \cos ^{2} \varphi$ given in tables 1, 2, and 3, and the values of $R$ were calculated from $F$ and $T$ using eq (6).

The values of $\Delta U$ thus calculated are in most cases only a small percentage of the measured values of $U$, so that the effect of air drag may be neglected. However, in the case of the 26.1 tex polyester yarn, the values of $\Delta U$ after $500 \mu$ s are sufficiently large that some correction to the $U, V$ data may be necessary. In estimating this correction it should be noted that the magnitude of $\Delta U$ is proportional to $t^{2}$, so that a plot of transverse wave front position as a function of time has a slight parabolic curvature. The velocity $U_{0}$, air drag neglected, is equal to the slope of the straight line tangent to this parabola at the origin. The value of $U$ actually measured will probably exceed $U_{0}$, but not to the extent indicated by the calculated values of $\Delta U$. The actual correction to be made is therefore a matter of judgment.

The two polyester yarns have similar stress-strain curves, but according to table $4, \Delta U$ for the 124.3 tex yarn is approximately $1 / 7$ the value of $\Delta U$ for the 26.1 tex yarn. Thus impact tests on larger diameter yarns are less subject to air drag effects than are tests on smaller diameter yarns of the same material. This is in agreement with the theory, as the following argument shows.

TABLE 4. Increase in transverse wave front velocity due to air drag

\begin{tabular}{|c|c|c|c|c|c|c|}
\hline \multirow{3}{*}{$\begin{array}{l}\text { Impact } \\
\text { velocity } \\
V\end{array}$} & \multicolumn{6}{|c|}{$\begin{array}{l}\text { Increase in transverse wave front velocity } \\
\qquad U\end{array}$} \\
\hline & \multicolumn{3}{|c|}{$200 \mu \mathrm{s}$ after impact } & \multicolumn{3}{|c|}{$500 \mu \mathrm{s}$ after impact } \\
\hline & $\begin{array}{l}\text { Polyester } \\
26.1 \text { tex }\end{array}$ & $\begin{array}{l}\text { Polyester } \\
124.3 \text { tex }\end{array}$ & $\begin{array}{c}\text { Nylon } \\
1 \% 2 Z \text { twist }\end{array}$ & $\begin{array}{l}\text { Polyester } \\
26.1 \text { tex }\end{array}$ & $\begin{array}{l}\text { Polyester } \\
124.3 \text { tex }\end{array}$ & $\begin{array}{c}\text { Nylon } \\
11 / 2 Z \text { twist }\end{array}$ \\
\hline \begin{tabular}{c}
\multicolumn{2}{c}{$\mathrm{m} / \mathrm{s}$} \\
100 \\
200 \\
300 \\
400 \\
500
\end{tabular} & $\begin{array}{r}m / s \\
0.2 \\
.7 \\
1.1 \\
2.0 \\
3.4\end{array}$ & $\begin{array}{r}m / s \\
0.0 \\
.1 \\
.2 \\
.3 \\
.5\end{array}$ & $\begin{array}{r}m / s \\
0.0 \\
.1 \\
.3 \\
.6 \\
1.2\end{array}$ & $\begin{array}{r}m / s \\
0.9 \\
4.4 \\
6.7 \\
12.7 \\
21.1\end{array}$ & $\begin{array}{r}m / s \\
0.2 \\
.6 \\
1.0 \\
1.9 \\
3.1\end{array}$ & $\begin{array}{r}m / s \\
0.2 \\
.9 \\
2.0 \\
3.5 \\
7.5\end{array}$ \\
\hline
\end{tabular}

According to eqs (1), (2), (3), and (4) the velocities $U_{0}, \lambda_{0}$, and $W_{0}$, and the angle $\phi_{0}$ are specific quantities independent of the diameter of the yarn. However, according to eq (15), the force per unit length $F$ due to air drag is proportional to $d$. Since the tension $T$ in the yarn is proportional to $d^{2}$, it may be seen from eq (6) that the radius of curvature $R$ is proportional to $d$. Thus from eq (16) $\Delta U$ is inversely proportional to $d^{2}$. The tex value of a yarn is proportional to $d^{2}$, so that $\Delta U$ is inversely proportional to the tex. For the two polyester yarns the tex ratio is 5 to 1 .

\section{Summary}

McCrackin's theory on the effects of air drag has been tested by transverse impact experiments on two samples of high tenacity polyester yarn, 26.1 tex and 124.3 tex, and one sample of high tenacity nylon yarn, 93.3 tex. Impact velocities ranged between $100 \mathrm{~m} / \mathrm{s}$ and $400 \mathrm{~m} / \mathrm{s}$. In all of the tests curvature due to air drag was observed in the yarn configuration

As the length of yarn in the transverse wave increased with increasing time after impact, the radius of curvature increased, approaching a constant value consistent with that predicted by McCrackin's theory. The radius of curvature also decreased as the impact velocity was increased, in agreement with the theory. Results gave approximate agreement with the theoretical prediction that the radius of curvature is proportional to yarn diameter.

For tests at $500 \mathrm{~m} / \mathrm{s}$ impact velocity on the 26.1 tex polyester yarn, a small correction to the measured value of transverse wave front velocity was necessary in the calculation of an accurate stress-strain curve. For the other two yarns these corrections for the effect of air drag were negligible.

\section{References}

[1] D. R. Petterson, G. M. Stewart, F. A. Odell, and R. C. Maheux, Dynamic distribution of strain in textile materials under high-speed impact, Part I: Experimental methods and preliminary results on single yarns, Textile Research J. 30, 411-421 (June 1960).

[2] D. R. Petterson and G. M. Stewart, Dynamic distribution of strain in textile materials under high-speed impact, Part II: Stress-strain curves from strain position distributions, Textile Research J. 30, 422-431 (June 1960).

[3] M. N. Pilsworth, Tensile impact on rubber and nylon, Pioneering Research Division, Quartermaster Research and Engineering Center, Natick, Mass., Technical Report PR-3 (May 1962).

[4] J. C. Smith, C. A. Fenstermaker, and P. J. Shouse, The behavior of filamentous materials subjected to highspeed tensile impact, ASTM special technical publication No. 336, Symposium on the dynamic behavior of materials, 47-69 (1963).

[5] J. C. Smith, C. A. Fenstermaker, and P. J. Shouse, Stressstrain relationships in yarns subjected to rapid impact loading, Part X: Stress-strain curves obtained by impacts with rifle bullets, Textile Research J. 33, 919-934 (Nov. 1963).

[6] F. L. McCrackin, Effect of air drag on the motion of a filament struck transversely by a high-speed projectile, J. Research NBS 66C (Engr. and Instr.) No. 4, 317-322 (1962).

[7] R. B. Morrison and M. J. Ingle, Design data for aeronautics and astronautics (John Wiley \& Sons, Inc., New York, N.Y., 1962).

[8] S. F. Hoerner, Fluid dynamic drag (1958) published by S. F. Hoerner.

[9] Textile World (330 West 42d St., New York 36, N.Y.), 1962 Man-made fiber chart. 